\title{
World War II Effects on Prevalence and Pattern of Multimorbidity in Older Adults: Results From the KORA-Age Study
}

\author{
Ava Arshadipour ( $\square$ azam.arshadipour@helmholtz-muenchen.de ) \\ Helmholtz Zentrum München, German Research Center for Environmental Health \\ Barbara Thorand \\ Helmholtz Zentrum München, German Research Center for Environmental Health \\ Birgit Linkohr \\ Helmholtz Zentrum München, German Research Center for Environmental Health \\ Susanne Rospleszcz \\ Helmholtz Zentrum München, German Research Center for Environmental Health \\ karl-heinz Ladwig \\ Technical University of Munich \\ Margit Heier \\ Helmholtz Zentrum München, German Research Center for Environmental Health \\ Annette Peters \\ Helmholtz Zentrum München, German Research Center for Environmental Health
}

\section{Research Article}

Keywords: Chronic disease, Multimorbidity, Geriatrics, logistic regression, Machine learning, Agglomerative hierarchical clustering

Posted Date: October 22nd, 2021

DOl: https://doi.org/10.21203/rs.3.rs-326222/v2

License: (ㅇ) (1) This work is licensed under a Creative Commons Attribution 4.0 International License. Read Full License 


\section{Abstract}

\section{Background}

While risk factors for age-related diseases may increase multimorbidity (MM), early life deprivation may also accelerate the development of chronic diseases and MM.

\section{Methods}

This study explores the prevalence and pattern of MM in 65-71 year-old individuals born before, during, and after World War II in Southern Germany based on two KORA (Cooperative Health Research in the Region of Augsburg) -Age studies. MM was defined as having at least two chronic diseases, and birth periods were classified into five phases: pre-war, early war, late war, famine, and after the famine period. Logistic regression models were used to analyze the effect of the birth phases on MM with adjustment for sociodemographic and lifestyle risk factors. Furthermore, we used agglomerative hierarchical clustering to investigate the co-occurrence of diseases.

\section{Results}

Participants born during the late war phase had the highest prevalence of MM (62.2\%) and single chronic diseases compared to participants born during the other phases. Being born in the late war phase was significantly associated with a higher odds of $\mathrm{MM}(\mathrm{OR}=1.83,95 \% \mathrm{Cl}$ : $1.15-2.91)$ after adjustment for sociodemographic and lifestyle factors. In women, the prevalence of joint, gastrointestinal, eye diseases, and anxiety was higher, while heart disease, stroke, and diabetes were more common in men. Moreover, three main chronic disease clusters responsible for the observed associations were identified: joint and psychosomatic, cardiometabolic and, internal organs diseases.

\section{Conclusions}

Our findings imply that adverse early-life exposure may increase the risk of MM in adults aged 65-71 years. Moreover, identified disease clusters are not coincidental and require more investigation.

\section{Introduction}

Parallel to the worldwide increase in life expectancy in the last decades, the prevalence of age-related chronic diseases has also risen. Consequently, multimorbidity (MM), defined as the presence of at least two chronic diseases has become increasingly prevalent especially among older adults. MM is a major concern in the health care system since it can result in reduced quality of life, increased mortality, disability, and higher health care costs [1].

Based on a systematic literature review of 41 articles from different countries, the prevalence of MM ranges from 55-98\% in those aged $\geq 65$ years [2]. In Germany, based on the cross-sectional national telephone health interview survey "German Health Update" (GEDA 2012-2013), the MM prevalence ranged from $61.7 \%$ (95\% Cl: 59.3 -64.1) for 60 to 69 year-old to $72.9 \%$ (95\% Cl: 70.4-75.2) for 70 to 79 year-old individuals [3]. Others reported a $62 \%$ MM prevalence for those aged $\geq 65$ years in the German population [4]. In Augsburg, MM prevalence was 58.6\% for individuals aged 65-94 years based on the KORA-Age 1 data in 2008 [5]. Various studies have assessed the association of MM with sociodemographic and lifestyle factors, which indicated MM has been mainly associated with age, sex, educational level, income, physical activity, smoking, and alcohol consumption [6, 7].

Studies have suggested childhood adversity as a potential exposure that may increase MM risk at older age. Socioeconomic hardship and traumatic events such as abuse and neglect during early life stages can contribute to chronic diseases progression [8, 9]. Regarding the early life experience, it is also worth noting that there were widespread food shortages during World War Il, especially in the Soviet Union, India, China, Java, Vietnam, Greece, Austria, and Netherland. However, in Germany, food shortages mainly occurred at the end of World War II due to the destruction of agricultural land, livestock, machinery, and labor shortages. The average energy consumption per person decreased from about 2500 calories in 1944 to $1050-1250$ calories in 1946 in Germany, and after June 1948, it increased to 1800 calories again due to the currency reform [10]. Since aged adults in our sample born in Germany from 1937 to 1950 were exposed to this crucial period in their early phases of life, it offers the opportunity to study how the adversity of Word War II affected the prevalence of MM and chronic diseases later in life.

The potential clustering of chronic diseases is in particular relevant for prevention, diagnosis, and treatment. Various studies have explored different approaches to determine the co-occurrence of chronic diseases, such as analyzing the prevalence of specific disease combinations, network analysis, factor analysis, and multiple correspondence analysis [11]. Kirchberger et al. [5] used factor analysis based on the Tetrachoric correlation matrix for 4127 persons. They identified four main comorbidity patterns: metabolic/ cardiovascular diseases, liver/lung/joint/eye diseases,

anxiety/depression/neurologic diseases, and cancer/gastrointestinal problems. To specify the chronic diseases clustering, only a few studies to date used clustering as an unsupervised machine learning algorithm, which has some advantages: No initial assumption on data distribution, the number of clusters or clusters structure is needed in most algorithms and the results are very informative by using dendrograms for visualization [11].

Therefore, we aimed to determine the effect of adverse developmental age situations on MM development and single chronic diseases in older individuals aged 65-71 years. In addition, we explored the co-occurrence of chronic diseases to specify the clusters of these chronic diseases in this age group. 


\section{Methods}

\section{Data collection and study sample}

Data originated from two population-based cross-sectional KORA-Age study arms: KORA-Age 1 conducted from 01.12.2008 to 06.11.2009 and KORA-Age 3 conducted from 01.02.2016 to 07.10.2016, which are follow-ups of four independent cross-sectional studies (S1 1984/5, S2 1989/90, S3 1994/5, and S4 1999-2001). Both studies focused on the health of participants aged 65 and older in Augsburg and the two adjacent regions in Southern Germany based on questionnaires and telephone interviews. The KORA-Age study is described in detail elsewhere [12]. In short, 4,565 out of 5,991 eligible individuals (response rate: 76.2\%) participated in the survey, and 1,920 were between 65 and 71 year-old on 31.12 .2008 (born between 1937-1943). The KORA-Age 3 survey consisted of 4,083 out of 6,051 eligible individuals (response rate: $67.5 \%$ ), of whom 1,444 participants were in the same age range on 31.12.2015 (born between 1944-1950). The present analysis combines these two KORA-Age study arms, which were completely independent because of the different birth years (Figure 1).

\section{Exposure: Birth phases}

The exposure variable was defined based on the participants' crucial developmental age during prenatal gestation or the first two years of life. Based on the World War II situation in the Augsburg area, individuals were divided into five independent birth periods [Figure 1]: phase 1 (pre-war, January 1937August 1939), phase 2 (early war, September 1939-December 1943), phase 3 (late war, January 1944-April 1945), phase 4 (famine period after the war, May 1945- Jun 1948) and phase 5 (after famine period and reconstruction, July 1948-Dec 1950) [10].

\section{Outcome: Multimorbidity}

The primary outcome was MM, defined as the presence of two or more concomitant chronic diseases in individuals [5]. We considered fourteen major chronic diseases, including hypertension, eye disease, heart diseases, diabetes, joint disease, lung disease, gastrointestinal disease, stroke, cancer, kidney diseases, liver diseases, neurological diseases, depression, and anxiety. All disease variables were defined as life-time diagnoses except for cancer diagnosed within the last three years. Hypertension, diabetes, cancer, stroke, heart diseases (myocardial infarction and coronary artery disease) were assessed based on the questionnaire. All other diseases were identified in a telephone interview based on the Charlson Comorbidity Index [12]. Participants were asked whether they suffer from kidney, liver, lung diseases (e.g., asthma, chronic bronchitis, and emphysema), inflammatory joint problems (e.g., arthritis or rheumatism), gastrointestinal diseases (e.g., colitis, cholecystic, gastric, or ulcer), heart diseases (e.g., congestive heart failure, coronary heart failure, or angina), eye problem (e.g., cataract, retinitis pigmentosa, glaucoma, macular degeneration, diabetic retinopathy). Neurological diseases were evaluated based on diseases like epilepsy, Parkinson's, or sclerosis using telephone interviews. The Geriatric Depression Scale [13] and Generalized Anxiety Disorder Scale-7 [14] were used to diagnose depression and anxiety. Individuals with scores $\geq 10$ were defined as suffering from depression or anxiety.

\section{Explanatory variables}

We considered age, sex, education level, alcohol consumption, physical activity, body mass index (BMI), smoking behavior, and cognitive status as covariates. Education levels were based on the duration of education and vocational training and categorized into three groups: low (9 years or less), middle (10 or 11 years), and high (12 years or more). Body mass index (BMI), defined by the World Health Organization (WHO), was used. Participants were categorized as underweight or normal weight $\left(\mathrm{BMI}<24.99 \mathrm{~kg} / \mathrm{m}^{2}\right)$, overweight $(25 \leq \mathrm{BMI} \leq 29.99)$, obese class I (30 $\left.\leq \mathrm{BMI}<34.99\right)$, and obese class II or III (35 $\leq$ BMI) [15].

Leisure time physical activity was measured from two separate questions about leisure time sports activity in winter and summer, including cycling. Possible answers were (1) $>2$ hours, (2) $1-2$ hours, (3) $<1$ hour and (4) none. Participants, who had a total score less than 5 , obtained by summing the numbers (1)-(4) relating to activities in winter and summer, were classified to be "physically active" [16].

Alcohol consumption was based on self-reported alcohol intake with the following five groups: "almost every day", "several times a week", "about once a week", "less than once a week", and "never or seldom" [17]. For our analysis, we categorized the first two groups as "daily use" and the last two as "never or rare use." Based on self-reported information, there are three categories for smoking status: never smokers, former smokers, and active smokers. The cognitive status is identified as dementia, mildly impaired cognitive status, and normal status based on TICS-M (Telephone Interview for Cognitive Status) score, a standard instrument for assessing cognitive impairment [18].

\section{Statistical Analysis}

The frequency and prevalence of baseline characteristics were stratified by sex and birth phases, and the Chi-squared test was computed to check the differences. Overall, the stratified prevalence of MM and single diseases was calculated and tested by the Chi-squared test. Then Post-hoc tests with Bonferroni adjustment were performed for multiple comparisons. Covariates multi-collinearity was assessed using the variance inflation factors (VIF). Associations of the birth phases and MM were estimated by odds ratios (OR) in logistic regression models with different adjustment steps for risk factors. The Modeling process started with the standardized age variable as covariate only (model 1), then birth period only (model 2), standardized age (rescaled with mean and standard deviation) and birth period together (model 3), then sex, education, alcohol use, physical activity, BMI, smoking behavior and cognitive status were added to the final model (models 4). We used a standardized age variable to prevent potential bias because of different age distributions in the different birth phases. The interaction effect of sex and birth phase variable also was checked in the final model. Agglomerative hierarchical clustering approach as an unsupervised machine learning technique was carried out to identify disease clusters so that 
diseases in one cluster are more similar than diseases in other clusters. This bottom-up algorithm begins with each disease as an individual cluster and merges the similar clusters until remaining only one cluster based on the proximity distance matrix. The average linkage method as proximity distance and Yule Q coefficient as similarity measurement for the binary disease variables were considered. The final cluster selection was created based on the threshold (cutoff height), corresponding to subject information, prior research, and clinical significance.

Since there is a big difference between the prevalence of hypertension and other chronic diseases, regression models and cluster analysis were performed without hypertension as a sensitivity analysis. Furthermore, the Ward and Single linkage methods, as other possible determinants for the pairwise distance between the set of observations were used to determine the robustness of agglomerative hierarchical clustering [11].

\section{Results}

\section{Study population characteristics}

The final sample consisted of 3377 participants aged 65 to 71 years (Figure1). From this population, 684 persons (52.2\% female) were born in pre-war, 1236 persons ( $50.8 \%$ female) in early war, 283 persons ( $51.9 \%$ female) in the late war, 574 persons (53.5\% female) in famine period, and 600 persons (53.7\% female) in after famine phase.

The overall baseline characteristics of the participants and their stratification by each phase are displayed in Table 1 . Men were more likely to have a high educational level $(45.0 \%$ versus $19.7 \%, p<0.001)$, drink alcohol daily $(65.1 \%$ versus $29.8 \%, p<0.001)$, have pre-obesity $(52.1 \%$ versus $38.9 \%$, $p<0.001)$ or obesity class I $(20.4 \%$ versus $17.0 \%, \mathrm{p}<0.001)$, be a former smoker $(57.5 \%$ versus $32.2 \%, \mathrm{p}<0.001)$ and have a slightly impaired cognitive status $(8.7 \%$ versus $4.8 \%, \mathrm{p}<0.001$ ) compared with women. 
Table 1

Descriptive statistics Percentages stratified by sex and birth phases for the whole participants ( $n=3,377)$ from KORA-Age 1 and KORA-Age 3 .

\begin{tabular}{|c|c|c|c|c|c|c|c|c|c|c|c|}
\hline \multirow[t]{2}{*}{ Characteristic } & & \multirow{2}{*}{$\begin{array}{l}\text { Total } \\
(\mathrm{N}=3,377)\end{array}$} & \multicolumn{3}{|l|}{ Sex } & \multicolumn{6}{|c|}{ Birth phases } \\
\hline & & & $\begin{array}{l}\text { Male } \\
(\mathrm{N}=1,616)\end{array}$ & $\begin{array}{l}\text { Female } \\
(\mathrm{N}=1,761)\end{array}$ & $\begin{array}{l}\mathrm{P}- \\
\text { value }\end{array}$ & $\begin{array}{l}\text { Phase } 1 \\
(\mathrm{~N}=684)\end{array}$ & $\begin{array}{l}\text { Phase 2 } \\
(\mathrm{N}=1,236)\end{array}$ & $\begin{array}{l}\text { Phase } 3 \\
(\mathrm{~N}=283)\end{array}$ & $\begin{array}{l}\text { Phase } 4 \\
(\mathrm{~N}=574)\end{array}$ & $\begin{array}{l}\text { Phase } 5 \\
(\mathrm{~N}=600)\end{array}$ & $\begin{array}{l}\mathrm{P}- \\
\text { value }\end{array}$ \\
\hline \multirow[t]{2}{*}{ Sex } & Male & 47.8 & $\ldots$ & $\ldots$ & \multirow[t]{2}{*}{$\ldots$} & 47.8 & 49.2 & 48.1 & 46.5 & 46.3 & \multirow[t]{2}{*}{0.76} \\
\hline & Female & 52.2 & $\ldots$ & $\ldots$ & & 52.2 & 50.8 & 51.9 & 53.5 & 53.7 & \\
\hline \multirow[t]{7}{*}{ Age } & 65 & 14.4 & 15.2 & 13.6 & \multirow[t]{7}{*}{0.371} & 0.0 & 20.7 & 0.0 & 0.0 & 38.3 & \multirow[t]{7}{*}{$<0.001$} \\
\hline & 66 & 14.7 & 15.5 & 13.8 & & 0.0 & 20.2 & 0.0 & 0.0 & 41.0 & \\
\hline & 67 & 15.0 & 14.4 & 15.6 & & 0.0 & 24.1 & 0.0 & 14.9 & 20.0 & \\
\hline & 68 & 15.5 & 14.6 & 16.4 & & 0.0 & 25.3 & 0.0 & 37.1 & 0.0 & \\
\hline & 69 & 14.8 & 14.9 & 14.6 & & 28.2 & 9.7 & 0.0 & 32.4 & 0.0 & \\
\hline & 70 & 11.8 & 12 & 11.6 & & 35.8 & 0.0 & 22.9 & 15.5 & 0.0 & \\
\hline & 71 & 13.7 & 13.2 & 14.2 & & 35.9 & 0.0 & 77.1 & 0.0 & 0.0 & \\
\hline \multirow[t]{2}{*}{$\begin{array}{l}\text { Age mean } \\
(\mathrm{SD})\end{array}$} & $\mathrm{MM}=$ yes & $68.1(1.9)$ & $68.1(2.0)$ & $68.2(1.9)$ & 0.325 & $\begin{array}{l}70.1 \\
(0.8)\end{array}$ & $66.9(1.3)$ & $\begin{array}{l}70.7 \\
(0.42)\end{array}$ & $\begin{array}{l}68.5 \\
(0.9)\end{array}$ & $\begin{array}{l}65.9 \\
(0.7)\end{array}$ & 0.750 \\
\hline & $\mathrm{MM}=$ no & $67.7(1.9)$ & $67.7(1.9)$ & $67.7(1.9)$ & 0.364 & $\begin{array}{l}70.1 \\
(0.7)\end{array}$ & $66.8(1.2)$ & $\begin{array}{l}70.7 \\
(0.4)\end{array}$ & $\begin{array}{l}68.5 \\
(0.9)\end{array}$ & $\begin{array}{l}65.8 \\
(0.7)\end{array}$ & 0.734 \\
\hline \multirow[t]{3}{*}{ Education } & Low & 10.2 & 3.2 & 16.6 & \multirow[t]{3}{*}{$<0.001$} & 16.5 & 11.6 & 5.6 & 5.4 & 6.7 & \multirow[t]{3}{*}{$<0.001$} \\
\hline & Middle & 58.0 & 51.8 & 63.7 & & 57.7 & 58.5 & 56.9 & 59.7 & 56.2 & \\
\hline & High & 31.7 & 45.0 & 19.7 & & 25.7 & 29.8 & 37.1 & 34.8 & 37.0 & \\
\hline \multirow[t]{3}{*}{$\begin{array}{l}\text { Alcohol } \\
\text { consumption }\end{array}$} & $\begin{array}{l}\text { Never or } \\
\text { rare use }\end{array}$ & 38.5 & 20.9 & 53.8 & \multirow[t]{3}{*}{$<0.001$} & 38.6 & 37.1 & 38.2 & 40.9 & 36.7 & \multirow[t]{3}{*}{0.038} \\
\hline & $\begin{array}{l}\text { Once a } \\
\text { week }\end{array}$ & 15.1 & 13.9 & 16.3 & & 12.4 & 15.1 & 20.5 & 13.7 & 17.2 & \\
\hline & Daily use & 46.7 & 65.1 & 29.8 & & 49.0 & 47.8 & 41.3 & 44.9 & 45.8 & \\
\hline \multirow{2}{*}{$\begin{array}{l}\text { Physical } \\
\text { Activity }\end{array}$} & Active & 66.8 & 69.2 & 64.6 & \multirow[t]{2}{*}{0.005} & 64.5 & 66.6 & 63.6 & 67.1 & 71.3 & \multirow[t]{2}{*}{0.072} \\
\hline & Inactive & 33.2 & 30.8 & 35.4 & & 35.5 & 33.3 & 36.4 & 32.7 & 28.7 & \\
\hline \multirow[t]{4}{*}{ BMI } & $\begin{array}{l}\text { Underweight } \\
\text { or normal } \\
\text { weight }\end{array}$ & 29.1 & 22.4 & 35.4 & \multirow[t]{4}{*}{$<0.001$} & 26.9 & 27.5 & 32.9 & 29.9 & 32.5 & \multirow[t]{4}{*}{$<0.001$} \\
\hline & Pre obese & 45.2 & 52.1 & 38.9 & & 48.2 & 47.1 & 46.2 & 41.0 & 41.5 & \\
\hline & $\begin{array}{l}\text { Obese class } \\
\text { I }\end{array}$ & 18.6 & 20.4 & 17.0 & & 19.9 & 19.6 & 12.0 & 19.3 & 17.5 & \\
\hline & $\begin{array}{l}\text { Obesity } \\
\text { class II or III }\end{array}$ & 6.2 & 4.4 & 7.9 & & 4.2 & 5.3 & 6.7 & 8.4 & 8.0 & \\
\hline \multirow{3}{*}{$\begin{array}{l}\text { Smoking } \\
\text { behavior }\end{array}$} & Never & 45.3 & 31.6 & 58 & \multirow[t]{3}{*}{$<0.001$} & 55.9 & 45.4 & 43.8 & 39.7 & 39.0 & \multirow[t]{3}{*}{$<0.001$} \\
\hline & $\begin{array}{l}\text { Active } \\
\text { smoker }\end{array}$ & 10.3 & 10.9 & 9.8 & & 7.3 & 10.1 & 10.6 & 9.4 & 15.0 & \\
\hline & $\begin{array}{l}\text { Former } \\
\text { smoker }\end{array}$ & 44.3 & 57.5 & 32.2 & & 36.8 & 44.3 & 45.6 & 50.9 & 46.0 & \\
\hline \multirow{3}{*}{$\begin{array}{l}\text { Cognitive } \\
\text { status }\end{array}$} & Good & 89.5 & 86.5 & 92.3 & $<0.001$ & 84.8 & 90.8 & 86.2 & 92.0 & 91.5 & $<0.001$ \\
\hline & $\begin{array}{l}\text { Mildly } \\
\text { impaired }\end{array}$ & 6.6 & 8.7 & 4.8 & & 10.0 & 5.6 & 8.1 & 5.2 & 5.7 & \\
\hline & Impaired & 2.1 & 2.8 & 1.5 & & 3.0 & 2.3 & 2.8 & 1.4 & 1.2 & \\
\hline Baseline & $\mathrm{S} 1$ & 26.0 & 25.3 & 26.7 & 0.685 & 27.6 & 26.8 & 24.0 & 24.8 & 24.7 & 0.17 \\
\hline $\begin{array}{l}\text { Values repre } \\
\text { variable and } \\
\text { (prenatal ges } \\
\text { Phase 4: Far }\end{array}$ & $\begin{array}{l}\% \text { unless ott } \\
\text { 7NOVA tes } \\
\text { on or the firs } \\
\text { Phase } 5: \text { Af }\end{array}$ & $\begin{array}{l}\text { vise indic } \\
\text { r compari } \\
\text { o years o } \\
\text { famine. }\end{array}$ & $\begin{array}{l}\text { the Palue } \\
\text { the mean } \\
\text { e) and the }\end{array}$ & $\begin{array}{l}\text { Chi-squa } \\
\text { ultiple ph } \\
\text { Id War II }\end{array}$ & $\begin{array}{l}\text { est for } \\
\text { s. Pha } \\
\text { tion in }\end{array}$ & $\begin{array}{l}\text { tegorica } \\
\text { s were d } \\
\text { ermany. }\end{array}$ & $\begin{array}{l}\text { riables, fr } \\
\text { ed based } \\
\text { ase 1: pre }\end{array}$ & $\begin{array}{l}\text { t-Test fo } \\
\text { varticipa } \\
\text {; Phase }\end{array}$ & $\begin{array}{l}\text { mparing } \\
\text { critical c } \\
\text { :arly War }\end{array}$ & $\begin{array}{l}\text { mean o } \\
\text { elopment } \\
\text { lase 3: L }\end{array}$ & $\begin{array}{l}\text { ge } \\
\text { Ware }\end{array}$ \\
\hline
\end{tabular}




\begin{tabular}{|c|c|c|c|c|c|c|c|c|}
\hline S2 & 23.5 & 23.4 & 23.6 & 21.1 & 25.0 & 25.1 & 23.2 & 22.5 \\
\hline S3 & 25.8 & 25.7 & 25.8 & 28.9 & 24.9 & 26.1 & 24.0 & 25.5 \\
\hline S4 & 24.7 & 25.6 & 24.0 & 22.4 & 23.2 & 24.7 & 28.0 & 27.3 \\
\hline
\end{tabular}

Values represent \% unless otherwise indicated. P-value from Chi-square test for categorical variables, from t-Test for comparing the mean of age variable and from ANOVA test for comparing the mean of multiple phases. Phases were defined based on participant's critical developmental age (prenatal gestation or the first two years of life) and the World War II situation in Germany. Phase 1: pre-War; Phase 2: Early War; Phase 3: Late War; Phase 4: Famine; Phase 5: After famine.

Educational levels increased over time with the lowest level of high education for people born before the war (26\% high education) and higher levels later (37\% high education in phase 5). Individuals born during and after the famine had a lower percentage $(41.0 \%$ and $41.5 \%$, p<0.001) of pre-obesity compared to the other phases. Participants born after the famine were more likely (15.0\%) to be active smokers than people born before. Individuals born during the late war phase had a higher percentage of mildly impaired (8.1\%) and impaired cognitive (2.8\%) status compared with other phases (Table 1$)$.

Prevalence of MM and single chronic diseases

MM prevalence was $49.4 \%$ in the total sample. There were no considerable differences in the prevalence of MM among men (48.8\%) and women (49.9\%) overall. MM prevalence was highest (62.2\%) for individuals born during phase 3 compared with the other phases (Table 2). In post hoc multiple comparisons, significant differences in MM prevalence were observed between phase 3 and phase 2 and between phase 3 and phase 5 . There was no difference in MM between men and women (Figure S1). Likewise, there were the same pattern in males and females and multiple comorbidities (Table S1). The prevalence of the chronic diseases stratified by sex and phases was presented in Table 2. Overall, hypertension (56.7\%), eye diseases (25.6\%), and heart diseases (18.9\%) were the most prevalent diseases in both men and women. Although, women had significantly higher prevalence of joint ( $13.1 \%$ vs. $7.4 \%, p<0.001)$, gastrointestinal (9.1\% vs. $6.7 \%, p=0.01)$, eye diseases $(28.5 \%$ vs. $22.5 \%, p<0.001)$ and anxiety $(7.8 \%$ vs. $3.4 \%, p<0.001)$, men had more heart diseases $(23.0 \%$ vs. $15.1 \%$, p<0.001), stroke $(6.8 \%$ vs. $3.1 \%, p<0.001)$ and diabetes $(17.1 \%$ vs. $12.9 \%$, $p<0.001)$. Furthermore, there was a significant difference in the prevalence of some single diseases according to the birth phases. For most diseases, the prevalence was highest among individuals born in phase 3 (Table 2). 
Table 2

Number and percentage of single diseases and multimorbidity stratified by sex and birth phases for the whole participants ( $n=3,377$ ) from KORA-Age 1 and KORA-Age 3 .

\begin{tabular}{|c|c|c|c|c|c|c|c|c|c|c|}
\hline \multirow[t]{2}{*}{ Disease } & \multirow{2}{*}{$\begin{array}{l}\text { Total } \\
(3,377)\end{array}$} & \multicolumn{3}{|l|}{ Sex } & \multicolumn{6}{|c|}{ Birth phases } \\
\hline & & $\begin{array}{l}\text { Male } \\
(1,616)\end{array}$ & $\begin{array}{l}\text { Female } \\
(1,761)\end{array}$ & $\begin{array}{l}\mathrm{P} \text { - } \\
\text { value }\end{array}$ & $\begin{array}{l}\text { Phase } 1 \\
(684)\end{array}$ & $\begin{array}{l}\text { Phase } 2 \\
(1,236)\end{array}$ & $\begin{array}{l}\text { Phase } 3 \\
\text { (283) }\end{array}$ & $\begin{array}{l}\text { Phase } 4 \\
\text { (574) }\end{array}$ & $\begin{array}{l}\text { Phase } 5 \\
(600)\end{array}$ & $\begin{array}{l}\mathrm{P} \text { - } \\
\text { value }\end{array}$ \\
\hline Multimorbidity (MM) & $\begin{array}{l}1667 \\
(49.4 \%)\end{array}$ & $\begin{array}{l}788 \\
(48.8 \%)\end{array}$ & $\begin{array}{l}879 \\
(49.9 \%)\end{array}$ & 0.503 & $\begin{array}{l}370 \\
(54.1 \%)\end{array}$ & $\begin{array}{l}561 \\
(45.4 \%)\end{array}$ & $\begin{array}{l}176 \\
(62.2 \%)\end{array}$ & $\begin{array}{l}310 \\
(54.0 \%)\end{array}$ & $\begin{array}{l}250 \\
(41.7 \%)\end{array}$ & $<0.001$ \\
\hline Lung & $\begin{array}{l}343 \\
(10.2 \%)\end{array}$ & $\begin{array}{l}157 \\
(9.7 \%)\end{array}$ & $\begin{array}{l}186 \\
(10.6 \%)\end{array}$ & 0.415 & $\begin{array}{l}61 \\
(8.9 \%)\end{array}$ & $\begin{array}{l}109 \\
(8.8 \%)\end{array}$ & $\begin{array}{l}35 \\
(12.4 \%)\end{array}$ & $\begin{array}{l}71 \\
(12.4 \%)\end{array}$ & $\begin{array}{l}67 \\
(11.2 \%)\end{array}$ & 0.065 \\
\hline $\begin{array}{l}\text { Joint diseases( Arthritis. } \\
\text { Rheumatic) }\end{array}$ & $\begin{array}{l}349 \\
(10.3 \%)\end{array}$ & $\begin{array}{l}119 \\
(7.4 \%)\end{array}$ & $\begin{array}{l}230 \\
(13.1 \%)\end{array}$ & $<0.001$ & $\begin{array}{l}74 \\
(10.8 \%)\end{array}$ & $\begin{array}{l}138 \\
(11.2 \%)\end{array}$ & $\begin{array}{l}31 \\
(11.0 \%)\end{array}$ & $\begin{array}{l}52 \\
(9.1 \%)\end{array}$ & $54(9.0 \%)$ & $<0.001$ \\
\hline Cancer & $\begin{array}{l}145 \\
(4.3 \%)\end{array}$ & $\begin{array}{l}74 \\
(4.6 \%)\end{array}$ & $71(4.0 \%)$ & 0.433 & $\begin{array}{l}25 \\
(3.7 \%)\end{array}$ & $55(4.4 \%)$ & $\begin{array}{l}13 \\
(4.6 \%)\end{array}$ & $\begin{array}{l}27 \\
(4.7 \%)\end{array}$ & $25(4.2 \%)$ & 0.898 \\
\hline Gastrointestinal & $270(8 \%)$ & $\begin{array}{l}109 \\
(6.7 \%)\end{array}$ & $\begin{array}{l}161 \\
(9.1 \%)\end{array}$ & 0.0101 & $\begin{array}{l}61 \\
(8.9 \%)\end{array}$ & $97(7.8 \%)$ & $\begin{array}{l}21 \\
(7.4 \%)\end{array}$ & $\begin{array}{l}49 \\
(8.5 \%)\end{array}$ & $42(7.0 \%)$ & 0.735 \\
\hline Heart diseases & $\begin{array}{l}637 \\
(18.9 \%)\end{array}$ & $\begin{array}{l}371 \\
(23 \%)\end{array}$ & $\begin{array}{l}266 \\
(15.1 \%)\end{array}$ & $<0.001$ & $\begin{array}{l}149 \\
(21.8 \%)\end{array}$ & $\begin{array}{l}216 \\
(17.5 \%)\end{array}$ & $\begin{array}{l}65 \\
(23.0 \%)\end{array}$ & $\begin{array}{l}121 \\
(21.1 \%)\end{array}$ & $\begin{array}{l}86 \\
(14.3 \%)\end{array}$ & $<0.001$ \\
\hline Stroke & $\begin{array}{l}165 \\
(4.9 \%)\end{array}$ & $\begin{array}{l}110 \\
(6.8 \%)\end{array}$ & $55(3.1 \%)$ & $<0.001$ & $\begin{array}{l}34 \\
(5.0 \%)\end{array}$ & $52(4.2 \%)$ & $\begin{array}{l}27 \\
(9.5 \%)\end{array}$ & $\begin{array}{l}32 \\
(5.6 \%)\end{array}$ & $20(3.3 \%)$ & $<0.001$ \\
\hline Kidney & $\begin{array}{l}117 \\
(3.5 \%)\end{array}$ & $\begin{array}{l}62 \\
(3.8 \%)\end{array}$ & $55(3.1 \%)$ & 0.257 & $\begin{array}{l}30 \\
(4.4 \%)\end{array}$ & $34(2.8 \%)$ & $\begin{array}{l}18 \\
(6.4 \%)\end{array}$ & $\begin{array}{l}24 \\
(4.2 \%)\end{array}$ & $11(1.8 \%)$ & 0.002 \\
\hline Liver & $\begin{array}{l}82 \\
(2.4 \%)\end{array}$ & $\begin{array}{l}43 \\
(2.7 \%)\end{array}$ & $39(2.2 \%)$ & 0.400 & $\begin{array}{l}15 \\
(2.2 \%)\end{array}$ & $29(2.3 \%)$ & $9(3.2 \%)$ & $\begin{array}{l}15 \\
(2.6 \%)\end{array}$ & $14(2.3 \%)$ & 0.913 \\
\hline Diabetes mellitus & $\begin{array}{l}504 \\
(14.9 \%)\end{array}$ & $\begin{array}{l}276 \\
(17.1 \%)\end{array}$ & $\begin{array}{l}228 \\
(12.9 \%)\end{array}$ & $<0.001$ & $\begin{array}{l}118 \\
(17.3 \%)\end{array}$ & $\begin{array}{l}165 \\
(13.3 \%)\end{array}$ & $\begin{array}{l}48 \\
(17.0 \%)\end{array}$ & $\begin{array}{l}95 \\
(16.6 \%)\end{array}$ & $\begin{array}{l}78 \\
(13.0 \%)\end{array}$ & 0.056 \\
\hline Neurological disease & $\begin{array}{l}103 \\
(3.1 \%)\end{array}$ & $\begin{array}{l}51 \\
(3.2 \%)\end{array}$ & $52(3.0 \%)$ & 0.731 & $\begin{array}{l}22 \\
(3.2 \%)\end{array}$ & $31(2.5 \%)$ & $\begin{array}{l}10 \\
(3.5 \%)\end{array}$ & $\begin{array}{l}20 \\
(3.5 \%)\end{array}$ & $20(3.3 \%)$ & 0.727 \\
\hline Hypertension & $\begin{array}{l}1914 \\
(56.7 \%)\end{array}$ & $\begin{array}{l}910 \\
(56.3 \%)\end{array}$ & $\begin{array}{l}1004 \\
(57.0 \%)\end{array}$ & 0.626 & $\begin{array}{l}396 \\
(57.9 \%)\end{array}$ & $\begin{array}{l}673 \\
(54.4 \%)\end{array}$ & $\begin{array}{l}179 \\
(63.3 \%)\end{array}$ & $\begin{array}{l}344 \\
(59.9 \%)\end{array}$ & $\begin{array}{l}322 \\
(53.7 \%)\end{array}$ & 0.013 \\
\hline Eye disease & $\begin{array}{l}866 \\
(25.6 \%)\end{array}$ & $\begin{array}{l}364 \\
(22.5 \%)\end{array}$ & $\begin{array}{l}502 \\
(28.5 \%)\end{array}$ & $<0.001$ & $\begin{array}{l}202 \\
(29.5 \%)\end{array}$ & $\begin{array}{l}285 \\
(23.1 \%)\end{array}$ & $\begin{array}{l}103 \\
(36.4 \%)\end{array}$ & $\begin{array}{l}164 \\
(28.6 \%)\end{array}$ & $\begin{array}{l}112 \\
(18.7 \%)\end{array}$ & $<0.001$ \\
\hline Anxiety & $\begin{array}{l}192 \\
(5.7 \%)\end{array}$ & $\begin{array}{l}55 \\
(3.4 \%)\end{array}$ & $\begin{array}{l}137 \\
(7.8 \%)\end{array}$ & $<0.001$ & $\begin{array}{l}55 \\
(8.0 \%)\end{array}$ & $87(7.0 \%)$ & $9(3.2 \%)$ & $\begin{array}{l}24 \\
(4.2 \%)\end{array}$ & $17(2.8 \%)$ & $<0.001$ \\
\hline Depression & $\begin{array}{l}44 \\
(1.3 \%)\end{array}$ & $\begin{array}{l}16 \\
(1.0 \%)\end{array}$ & $28(1.6 \%)$ & 0.131 & $\begin{array}{l}12 \\
(1.8 \%)\end{array}$ & $15(1.2 \%)$ & $3(1.1 \%)$ & $9(1.6 \%)$ & $5(0.8 \%)$ & 0.616 \\
\hline
\end{tabular}

P-value from Chi-square test for categorical variables. Phases were defined based on participant's critical developmental age (prenatal gestation or the first two years of life) and the World War II situation in Germany. Phase 1: pre-War; Phase 2: Early War; Phase 3: Late War; Phase 4: Famine; Phase 5: After famine.

\section{Association between birth phase and MM}

In the age-adjusted logistic regression model, the odds ratio of $\mathrm{MM}$ in phase $3(\mathrm{OR}=1.69,95 \% \mathrm{Cl}: 1.08-2.64)$ and phase $4(\mathrm{OR}=1.39,95 \% \mathrm{Cl}$ : $1.04-1.87)$ were significantly higher compared to the reference phase 5 . After adjusting for other covariates (model 4), participants born in phase 3 had a higher odds of $\mathrm{MM}$ compared with the individuals born in phase $5(\mathrm{OR}=1.83,95 \% \mathrm{Cl}$ : 1.15-2.91) (Table 3). We did not observe any significant interaction between sex and birth phase on the odds of MM (Figure S1). 
Table 3

Odds ratios and $95 \%$ confidence intervals for multimorbidity based on hierarchical logistic regression for the whole participants ( $\mathrm{n}=3,377$ ) from KORA-Age 1 and KORA-Age 3.

\begin{tabular}{|c|c|c|c|c|c|}
\hline Characteristics & & model 1 & model 2 & model 3 & model 4 \\
\hline Standardized age & & $1.12(1.08-1.16)$ & & $1.12(0.99-1.29)$ & $1.11(0.96-1.27)$ \\
\hline \multirow[t]{4}{*}{ Birth phases (ref: phase 5) } & Phase 1 & & $1.64(1.32-2.05)$ & $1.26(0.88-1.82)$ & $1.35(0.92-1.98)$ \\
\hline & Phase 2 & & $1.16(0.95-1.41)$ & $1.09(0.88-1.34)$ & $1.08(0.87-1.34)$ \\
\hline & Phase 3 & & $2.3(1.72-3.07)$ & $1.69(1.08-2.64)$ & $1.83(1.15-2.91)$ \\
\hline & Phase 4 & & $1.64(1.30-2.07)$ & 1.39 (1.04-1.87) & $1.35(0.99-1.84)$ \\
\hline Female (ref: male) & & & & & $0.98(0.83-1.16)$ \\
\hline \multirow[t]{2}{*}{ Education (ref: low) } & Middle & & & & $0.82(0.63-1.05)$ \\
\hline & High & & & & $0.72(0.55-0.95$ \\
\hline \multirow[t]{2}{*}{ Alcohol consumption (ref: never or rare use) } & Once a week & & & & $0.99(0.8-1.2)$ \\
\hline & Daily use & & & & $0.76(0.64-0.91)$ \\
\hline Physical Activity (active) & Inactive & & & & $1.47(1.26-1.72)$ \\
\hline \multirow[t]{3}{*}{ BMI (ref: underweight or normal) } & Overweight & & & & $1.32(1.11-1.57)$ \\
\hline & Obesity grade I & & & & $1.92(1.55-2.38)$ \\
\hline & Obesity grade II or III & & & & $3.09(2.21-4.33)$ \\
\hline \multirow[t]{2}{*}{ Smoking behavior (ref: never smoker) } & Active smoker & & & & $1.34(1.05-1.72)$ \\
\hline & Ex-smoker & & & & $1.36(1.16-1.59)$ \\
\hline \multirow[t]{2}{*}{ Cognitive status (ref: good) } & Mildly impaired & & & & $1.43(1.07-1.92)$ \\
\hline & Impaired & & & & $1.32(0.81-2.18)$ \\
\hline AIC & & 4638.4 & 4639.0 & 4637.8 & 4373.6 \\
\hline
\end{tabular}

\section{The pattem of comorbidity}

Three main clusters of diseases were specified based on the agglomerative hierarchical clustering. The first cluster composed of joint and psychosomatic disorders consisted of anxiety, depression, joint and neurological diseases. The second cluster of cardio-metabolic diseases comprised diabetes, hypertension, stroke, and heart diseases. The last one was the internal organ diseases cluster, including the lung, gastrointestinal, kidney, and liver diseases (Figure 2).

The number and percent of multimorbid individuals for each cluster were calculated by dividing the number of individuals who had at least two of these diseases in the cluster by the total number of multimorbid patients. In total, 1002 of the 1667 participants with MM could be assigned to at least one cluster. A high percentage (49.1\%) of multimorbid participants were assigned to the cardiometabolic cluster. The internal organ cluster had a similar prevalence in terms of sex and different phases of the birth period; however, the joint and psychosomatic diseases cluster had a more significant prevalence in women (6.4\%) and phase 1 (7.0\%) and phase 2 (6.1\%) of birth years. Moreover, men (57.9\%) and participants born in phase 1 (52.7\%) and phase 3 (52.8\%) had a significantly higher prevalence in the cardiometabolic disease cluster (Table 4). 
Table 4

Number and percent of individuals with multimorbitiy (i.e. at least two diseases) in each cluster

\begin{tabular}{|c|c|c|c|c|c|c|c|c|c|c|}
\hline \multirow[t]{2}{*}{ Cluster } & \multirow{2}{*}{$\begin{array}{l}\text { Total } \\
(1,667)\end{array}$} & \multicolumn{3}{|l|}{ Sex } & \multicolumn{6}{|c|}{ Birth phases } \\
\hline & & $\begin{array}{l}\text { Male } \\
(788)\end{array}$ & $\begin{array}{l}\text { Female } \\
(879)\end{array}$ & $\begin{array}{l}\mathrm{P} \text { - } \\
\text { value }\end{array}$ & $\begin{array}{l}\text { Phase } 1 \\
(370)\end{array}$ & $\begin{array}{l}\text { Phase } 2 \\
(561)\end{array}$ & $\begin{array}{l}\text { Phase } 3 \\
\text { (176) }\end{array}$ & $\begin{array}{l}\text { Phase } 4 \\
(310)\end{array}$ & $\begin{array}{l}\text { Phase } 5 \\
(250)\end{array}$ & $\begin{array}{l}\mathrm{P}- \\
\text { value }\end{array}$ \\
\hline $\begin{array}{l}\text { Joint. Neuro. Anxiety. } \\
\text { Depression }\end{array}$ & $\begin{array}{l}78 \\
(4.7 \%)\end{array}$ & $\begin{array}{l}22 \\
(2.8 \%)\end{array}$ & $56(6.4 \%)$ & $<0.001$ & $26(7 \%)$ & $34(6.1 \%)$ & $2(1.1 \%)$ & $9(2.9 \%)$ & $7(2.8 \%)$ & 0.002 \\
\hline $\begin{array}{l}\text { Heart. Stroke. Diabetes. } \\
\text { Hypertension }\end{array}$ & $\begin{array}{l}818 \\
(49.1 \%)\end{array}$ & $\begin{array}{l}456 \\
(57.9 \%)\end{array}$ & $\begin{array}{l}362 \\
(41.2 \%)\end{array}$ & $<0.001$ & $\begin{array}{l}195 \\
(52.7 \%)\end{array}$ & $\begin{array}{l}258 \\
(46 \%)\end{array}$ & $\begin{array}{l}93 \\
(52.8 \%)\end{array}$ & $\begin{array}{l}156 \\
(50.3 \%)\end{array}$ & $\begin{array}{l}116 \\
(46.4 \%)\end{array}$ & $<0.001$ \\
\hline $\begin{array}{l}\text { Lung. Gastro. Kidney. } \\
\text { Liver }\end{array}$ & $\begin{array}{l}106 \\
(6.4 \%)\end{array}$ & $\begin{array}{l}44 \\
(5.6 \%)\end{array}$ & $62(7.1 \%)$ & 0.218 & $20(5.4 \%)$ & $31(5.5 \%)$ & $17(9.7 \%)$ & $19(6.1 \%)$ & $19(7.6 \%)$ & 0.051 \\
\hline
\end{tabular}

\section{Sensitivity analysis}

We repeated the analysis without hypertension for $\mathrm{MM}$ and the findings were close to the previous model analysis including hypertension overall. Phase 3 still had the highest $\mathrm{MM}$ odds ratio (OR=2.14, 95\% Cl: 1.29-3.52). Moreover, phase 1 (OR=1.64, 95\% Cl: 1.07-2.49) and phase 4 (OR=1.52, 95\% Cl: 1.082.14) had a bit higher odds than hypertension consideration, and they were significant (Table S2). Hierarchical clustering was also performed without hypertension, and the three major clusters remained as before (Figure S5).

The dendrograms of chronic diseases association based on the Single and Ward linkage approaches were also very close to the average method. The number of main clusters and included diseases also remained stable (Figure S6, S7).

\section{Discussion}

\section{Multimorbidity}

In our study, the overall prevalence of MM was $49.4 \%$ for adults aged $65-71$ year-old. The comparison of the prevalence of MM between studies is hampered by differences in the examined age groups, included diseases and study areas, even in Germany [3]. While there was no difference between men and women in the MM prevalence in the present study like in other studies [4, 7], others observed a higher prevalence in women [19]. Although men had a higher prevalence of diabetes, heart disease, and stroke relative to women, the prevalence of joint, gastrointestinal, and eye diseases and anxiety was more remarkable for women in the present study.

\section{Multimorbidity according to birth phases}

The prevalence of MM and every single chronic disease was higher in phase 3. Since it is well known that MM and chronic diseases increase with increasing age [2], differences in the unadjusted prevalence could be biased by differences in the age distribution between birth phases 1 to 5 . Therefore, we also assessed the impact of the birth phase on MM in logistic regression models adjusted for age and other sociodemographic and lifestyle factors. We found that the OR of MM was significantly higher in phase 3 compared with phase 5 , even after adjusting for these potential confounders. This effect could be explained by the unfavorable living conditions were observed in the late war phase in the South Germany. Large parts of the city of Augsburg were devastated during the most extensive bombing raid at the end of February 1944 [20]. Participants born during the last years of war were thus exposed to food crises and famine in Germany in 1945 during early life stages [10]. In this context, it has previously been shown that maternal and earlylife malnutrition can negatively affect adults mental and physical health [21]. Moreover, an increased chronic health disease incidence was identified for aged individuals (born 1922-1960 in former West Germany) exposed to war during their utero and childhood [22]. These findings are important as they indicate that besides well-established risk factors in adult life, the birth phases and the living conditions during the World War II still are of concern today. Therefore, health service measures as well as individual treatment efforts may specifically need to pay attention to both men and women born 1944 to 1945.

\section{Clusters of chronic diseases}

We identified three major clusters with the clustering approach to recognize individuals with similar MM diseases. The internal organ cluster included illnesses involving main body organs, such as the stomach and intestines, kidneys, liver, and lung, which indicated the same co-occurrence in men and women and individuals born in different phases. Association among the lung, liver, and gastrointestinal diseases is consistent with Rodriguez-Roisin et al. [23] in adult patients based on the possible shared mechanisms. This coexistence might increase vascular diseases, such as POPH and HPS, and other chronic respiratory diseases coexisting with chronic hepatic diseases. Still, more research is warranted to corroborate our understanding and underlying mechanisms for this cluster.

We verified the association between joint diseases and psychosomatic disorders within the second cluster, more prevalent in women. Previous studies have established links between rheumatoid arthritis, mood disturbances, and neurological diseases [24, 25]. Lee et al. [24] observed the coexistence of 
Parkinson's disease and rheumatics. Lwin et al. [25] also observed that depression was twice as prevalent in patients with rheumatoid arthritis as in the general population.

The cardiometabolic cluster had the highest proportion of co-occurrence of cardiovascular and metabolic diseases, typically found in aged people, and it was more prevalent in men. This relationship also has been widely illustrated in prior populations [5, 26]. Sowers et al. [26] showed that hypertension prevalence was twice as high in people with diabetes than those without diabetes. Also, individuals with hypertension experienced diabetes more frequently than persons with normal blood pressure. They also reported that hypertension could be responsible for up to $75 \%$ of CVD in diabetes.

\section{Strengths and weaknesses}

One of our analysis strengths is that it was based on two large data sets from the KORA cohort study [27] with individuals born around World War II. This enabled us to analyze comorbidity and MM in different birth phases. The information also came from the specific age range of individuals from two KORA-Age studies that provided uniformity in data, which is essential for exploring disease patterns. This huge database also contained information about demographic, sociodemographic, physical, and mental health factors, which helped adjust a wide range of factors associated with MM. Another strength is that both cross-sectional KORA-Age studies used the same instruments in the interview; thus the data is less likely to be biased [28].

The clustering method helped discover disease comorbidity clusters that define specific risk domains and assign individuals to subgroups with common characteristics and risks. Furthermore, using of Yule Q coefficient enabled us to measure the correlation among the binary chronic disease data.

Our study does not come without its limitations. Although using the self-reported weight and measured height in the baseline information was economical and straightforward, it might underestimate the real value for BMI. Individuals mostly tend to report less weight, then the real value for BMI goes far from the reported one which might be subject to recall bias [29]. Moreover, since chronic disease prevalence was mainly based on self-reported data, disease severity was not considered. Furthermore, we only used the birth year of individuals. We did not have any information about childhood diet, mother's health or exposure to adversity, birth weight, separation of the child from the parent, and others, which may determine MM later in life. Since we examined the longitudinal association of birth phases with $\mathrm{MM}$, we identified the temporal sequence between exposure and outcome, which might support a potential causal link. To precludes any cause and effect interpretation, other covariates were simultaneously used in model adjustment.

\section{Conclusion}

This research offers insight into differences in the MM prevalence for individuals aged 65-71 years born in different periods before, during, and after World War II. Adverse circumstances experienced during the late war period may have contributed to the higher MM prevalence in adult life. Moreover, our findings suggest three main disease clusters: i. Joint and psychosomatic diseases (joint, neurological, anxiety, depression); ii. Cardiometabolic diseases (heart, stroke, diabetes, hypertension); iii. Internal organ diseases (lung, gastrointestinal, kidney, and liver). Although the adverse situation of World War II and famine may increase MM risk at retirement age, more comprehensive childhood and life course circumstances are required to explain long-term health consequences. Future research shall investigate potential interactions between risk factor profiles during adult life and adverse early life exposures on MM. Furthermore, our results on the diseases clustering focusing on three significant clusters of MM call for further investigation, specifically their association with genetic effects, environmental factors, and polypharmacy.

\section{Abbreviations}

MM: Multimorbidity

BMI: Body mass index

KORA: Cooperative Health Research in the Region of Augsburg

\section{Declarations}

\section{Funding}

The KORA study was initiated and financed by the Helmholtz Zentrum München - German Research Center for Environmental Health, funded by the German Federal Ministry of Education and Research (BMBF) State Bavaria. The KORA-Age project was financed by the German Federal Ministry of Education and Research (BMBF FKZ 01ET0713 and 01ET1003A) as part of the Health in the old-age program.

\section{Conflict of interest}

There were no conflicts of interest declared by the authors.

\section{Ethics Statement}

The Ethics Committee of the Bavarian Medical Association has approved the KORA-Age study (08094). Written informed consent was obtained from all study participants according to the Helsinki Declaration. 


\section{Informed consent}

All of the study's participants gave their informed consent.

\section{Acknowledgments}

The authors would like to thank the Augsburg participants and field staff who collaborated with the studies and the Helmholtz Zentrum München for their cooperation in preserving this complex data.

\section{Authors' contributions}

Conceptualization: AA; Methodology: AA; Statistical Analyses: AA; Evaluation and Interpretation: AA, AP, BT, SR, BL; Original Paper Draft: AA; Revision and Editing: AA, AP, BT, SR, BL, MH, KHL; Main Study Design: AP, BL, MH; Data Curation and Quality Assurance: AP, BL, MH; Supervision: AP; All authors have read and approved the final manuscript.

\section{Competing interests}

The authors declare that they have no competing interests.

\section{Availability of data and materials}

Data is not openly available, and participants' data privacy is protected by data-protection standards established by the ethics committee of Bavarian Chamber of Physicians, Munich. However, data could be available upon request through a project agreement with KORA (http://epi.helmholtzmuenchen.de/koragen/) and the KORA Board approval.

\section{References}

1. Quiñones AR, Markwardt S, Botoseneanu A. Multimorbidity Combinations and Disability in Older Adults. The Journals of Gerontology: Series A. 2016;71(6):823-30. doi:https://doi.org/10.1093/gerona/glw035.

2. Marengoni A, Angleman S, Melis R, et al. Aging with multimorbidity: A systematic review of the literature. Ageing Research Reviews. 2011;10(4):4309. doi:https://doi.org/10.1016/j.arr.2011.03.003

3. Puth M-T, Weckbecker K, Schmid M, Münster E. Prevalence of multimorbidity in Germany: impact of age and educational level in a cross-sectional study on 19,294 adults. BMC Public Health. 2017;17(1):826. doi:https://doi.org/10.1186/s12889-017-4833-3

4. van den Bussche $\mathrm{H}$, Schön G, Kolonko T, et al. Patterns of ambulatory medical care utilization in elderly patients with special reference to chronic diseases and multimorbidity-results from a claims data based observational study in Germany. BMC Geriatr. 2011;11:54doi:https://doi.org/10.1186/1471-2318-11-54

5. Kirchberger I, Meisinger C, Heier M, et al. Patterns of Multimorbidity in the Aged Population. Results from the KORA-Age Study. PLOS ONE. 2012;7(1):e30556. doi:https://doi.org/10.1371/journal.pone.0030556

6. Fabbri E, Zoli M, Gonzalez-Freire M, Salive ME, Studenski SA, Ferrucci L. Aging and Multimorbidity: New Tasks, Priorities, and Frontiers for Integrated Gerontological and Clinical Research. J Am Med Dir Assoc. 2015;16(8):640-7. doi:https://doi.org/10.1016/j.jamda.2015.03.013

7. Taylor AW, Price K, Gill TK, et al. Multimorbidity - not just an older person's issue. Results from an Australian biomedical study. BMC Public Health. 2010;10(1):718. doi:https://doi.org/10.1186/1471-2458-10-718

8. Tomasdottir MO, Sigurdsson JA, Petursson H, et al. Self Reported Childhood Difficulties, Adult Multimorbidity and Allostatic Load. A Cross-Sectional Analysis of the Norwegian HUNT Study. PloS one. 2015;10(6):e0130591-e. doi:https://doi.org/10.1371/journal.pone.0130591

9. Humphreys J, Jameson K, Cooper C, Dennison E. Early-life predictors of future multi-morbidity: results from the Hertfordshire Cohort. Age and Ageing. 2018;47(3):474-8. doi:https://doi.org/10.1093/ageing/afy005

10. Stephan A-J, Strobl R, Schwettmann L, et al. Being born in the aftermath of World War II increases the risk for health deficit accumulation in older age: results from the KORA-Age study. European Journal of Epidemiology. 2019;34(7):675-87. doi:https://doi.org/10.1007/s10654-019-00515-4

11. Ng SK, Tawiah R, Sawyer M, Scuffham P. Patterns of multimorbid health conditions: a systematic review of analytical methods and comparison analysis. International Journal of Epidemiology. 2018;47(5):1687-704. doi:https://doi.org/10.1093/ije/dyy134

12. Chaudhry S, Jin L, Meltzer D. Use of a Self-Report-Generated Charlson Comorbidity Index for Predicting Mortality. Medical Care. 2005;43(6):607-15.

13. Javaid I. Sheikh JAY. A knowledge assessment test for geriatric psychiatry. Hospital and community psychiatry. 1985;36:1160-6.

14. Spitzer RL, Kroenke K, Williams JBW, Löwe B. A Brief Measure for Assessing Generalized Anxiety Disorder: The GAD-7. Archives of Internal Medicine. 2006;166(10):1092-7. doi:https://doi.org/10.1001/archinte.166.10.1092

15. Obesity: preventing and managing the global epidemic Report of a WHO Consultation: WHO Technical Report Series 2000.

16. Perna L, Mielck A, Lacruz ME, et al. The association between resilience and diabetic neuropathy by socioeconomic position: Cross-sectional findings from the KORA-Age study. Journal of Health Psychology. 2013;20(9):1222-8. doi:https://doi.org/10.1177/1359105313510334

17. Röhrig N, Strobl R, Müller M, et al. Directed acyclic graphs helped to identify confounding in the association of disability and electrocardiographic findings: results from the KORA-Age study. Journal of Clinical Epidemiology. 2014;67(2):199-206. doi:https://doi.org/10.1016/j.jclinepi.2013.08.012 
18. Lacruz M, Emeny R, Bickel H, Linkohr B, Ladwig K. Feasibility, internal consistency and covariates of TICS-m (telephone interview for cognitive statusmodified) in a population-based sample: findings from the KORA-Age study. Int J Geriatr Psychiatry. 2013;28(9):971-8.

doi:https://doi.org/10.1002/gps.3916

19. King DE, Xiang J, Pilkerton CS. Multimorbidity Trends in United States Adults, 1988-2014. The Journal of the American Board of Family Medicine. 2018;31(4):503. doi:https://doi.org/10.3122/jabfm.2018.04.180008

20. Pöhlmann M. Es war gerade, als würde alles bersten. Munich, editor. Munich: Volk Verlag; 2019.

21. Lumey LH, Stein AD, Susser E. Prenatal Famine and Adult Health. Annual Review of Public Health. 2011;32(1):237-62. doi:https://doi.org/10.1146/annurev-publhealth-031210-101230

22. Akbulut-Yuksel M. War during childhood: The long run effects of warfare on health. Journal of Health Economics. 2017;53:117-30. doi:https://doi.org/10.1016/j.jhealeco.2017.02.005

23. Rodriguez-Roisin R, Bartolome SD, Huchon G, Krowka MJ, Inflammatory bowel diseases, chronic liver diseases and the lung, European Respiratory Journal. 2016; 47 (2): 638650. DOI: 10.1183/13993003.00647-2015

24. Lee K-W, Chien C-F, Wu M-N, Lai C-L, Liou L-M. Parkinsonism with newly diagnosed flare-up rheumatoid arthritis mimicking progressive supranuclear palsy. Neurol India. 2020;68(2):481-2. doi:https://doi.org/10.4103/0028-3886.284382

25. Lwin MN, Serhal L, Holroyd C, Edwards CJ. Rheumatoid Arthritis: The Impact of Mental Health on Disease: A Narrative Review. Rheumatol Ther. 2020;7(3):457-71. doi:https://doi.org/10.1007/s40744-020-00217-4

26. Sowers JR, Epstein M, Frohlich ED. Diabetes, hypertension, and cardiovascular disease: an update. Hypertension. 2001;37(4):1053-9. doi:https://doi.org/10.1161/01.hyp.37.4.1053

27. Peters A, Döring A, Ladwig KH, et al. [Multimorbidity and successful aging: the population-based KORA-Age study]. Z Gerontol Geriatr. 2011;44 Suppl 2:41-54. doi:https://doi.org/10.1007/s00391-011-0245-7

28. Pannucci CJ, Wilkins EG. Identifying and avoiding bias in research. Plast Reconstr Surg. 2010;126(2):619-25. doi:https://doi.org/10.1097/PRS.0b013e3181de24bc

29. Skeie G, Mode N, Henningsen M, Borch KB. Validity of self-reported body mass index among middle-aged participants in the Norwegian Women and Cancer study. Clin Epidemiol. 2015;7:313-23. doi:https://10.2147/CLEP.S83839

\section{Figures}

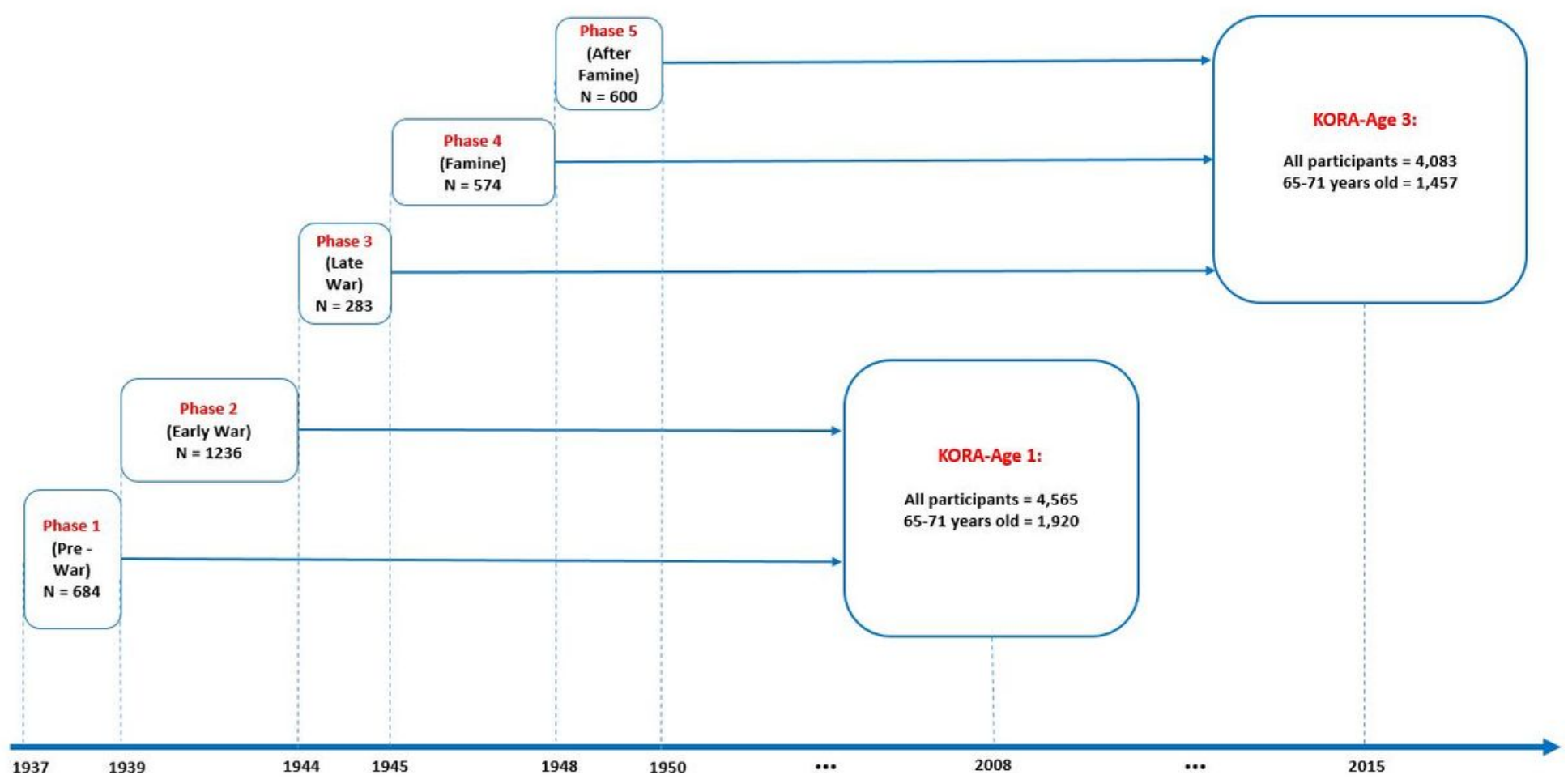

Figure 1

Participants of the KORA-Age 1 and KORA-Age 3. Combined study population ( $N=3,377)$ and birth phases. 2008 and 2015 referred to the years for the age calculation in two studies. Phases were defined based on participants critical developmental age (prenatal gestation or the first two years of life) and the World War II situation in Germany. 


\section{Cluster Dendrogram}
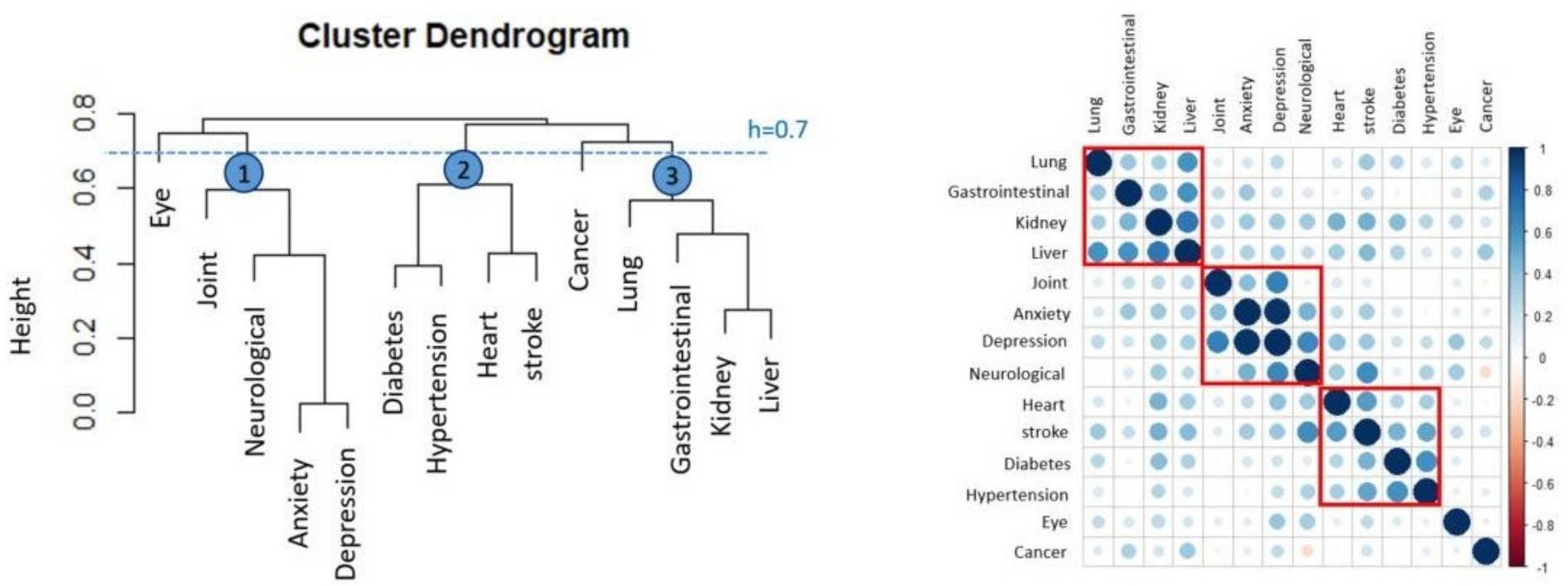

Figure 2

Left: Dendrogram based on the hierarchical clustering of chronic diseases using the average linkage method and Yule-Q coefficient. Right: Correlation matrix of chronic diseases based on Yule coefficient.

\section{Supplementary Files}

This is a list of supplementary files associated with this preprint. Click to download.

- supplementaryBMCBT.docx 